\title{
Molecular identification and expression of two non-P450 enzymes, monoamine oxidase $A$ and flavin-containing monooxygenase 2 , involved in phase I of xenobiotic biotransformation in the Pacific oyster, Crassostrea gigas
}

\author{
Isabelle Boutet ${ }^{a}$, Arnaud Tanguy $^{b}$ and Dario Moraga ${ }^{b_{*}}$,
}

\author{
a Station Méditerranéenne de l'Environnement Littoral, UMR CNRS-IFREMER 5171 "Génome, Populations, \\ Interactions, Adaptation", 1 Quai de la Daurade, 34200 Sète, France \\ ${ }^{b}$ Laboratoire des Sciences de I'Environnement Marin (LEMAR), UMR-CNRS 6539, Institut Universitaire Européen \\ de la Mer, Université de Bretagne Occidentale, Technopole Brest Iroise, Place Nicolas Copernic, Plouzané, F- \\ 29280, France \\ * Dario.Moraga@univ-brest.fr Tel.: +33-2-98-49-86-42; fax: +33-2-98-49-86-45.
}

\begin{abstract}
Marine bivalve metabolism can be perturbed by hydrocarbon and pesticide pollution in coastal ecosystems. In this study, in the Pacific oyster, Crassostrea gigas, full-length cDNAs encoding two non-P450 phase I enzymes, flavin-containing monooxygenase 2 (FMO-2) and monamine oxidase A (MAO A), were characterized. Both sequences contained the co-factor fixation motifs characteristic of their respective enzyme families. Using reverse transcription polymerase chain reaction (RT-PCR), the messenger RNA (mRNA) transcription levels of these two enzymes in tissues of oysters exposed, under experimental conditions, to hydrocarbons and two pesticide treatments were investigated. The pesticide treatments were exposure to either glyphosate or to a mixture composed of atrazine, diuron and isoproturon. The results showed a strong differential expression of FMO-2 and MAO A that was both tissue-specific as well as time- and treatment-dependent. It was also clearly demonstrated that the transcription levels of MAO A (generally considered a constitutive enzyme without external regulation) were induced by hydrocarbons and pesticides in digestive gland and inhibited by pesticides in gill tissue. Furthermore, the transcription levels of FMO-2 and MAO A mRNA in digestive gland might be useful as a marker of hydrocarbon or pesticide exposure in monitoring programs.
\end{abstract}

Keywords: Monoamine oxidase A; Flavin-containing monooxygenase 2; Crassostrea gigas 


\section{Introduction}

Marine organisms are continuously exposed to a wide variety of anthropogenic contaminants from industry, agriculture or urban effluents. The marine organisms, and especially the Pacific oyster Crassostrea gigas, living in coastal ecosystems have developed defence mechanisms against xenobiotic contamination, such as stress proteins (molecular chaperoning) (Boutet et al., 2003), metallothioneins (metal detoxification) (Tanguy et al., 2001; Tanguy and Moraga, 2001; Tanguy et al., 2002; Boutet et al., 2002), biotransformation enzyme system (organic compounds detoxification) (Boutet et al., in press), etc. The biotransformation system essentially involved enzymes capable of transform organic compounds in more soluble and easily excreted molecules than parent compound. These mechanism is divided into three phases. The first one is characterised by the oxygenation of the organic compound via microsomal monooxygenase enzymes, among them the cytochrome P450 pathway constitute the major detoxification system, followed by flavincontaining monooxygenase (FMO; Schlenk, 1998), monoamine oxidase (MAO; Strolin Benedetti, 2001), oxidase molybdenum hydroxylase (aldehyde and xanthine oxidase; Beedham, 1997) and alcohol and aldehyde dehydrogenase (Beedham, 1997). The resulting products are either directly excreted or more reactive than the parent product, entailing cell damages (DNA, lipids, protein) (Michel et al., 1992). The second phase is characterised by the enzymatic conjugation of phase I resulting products to endogenous compounds via glutathione S-transferases (GSTs), UDPglucuronyl transferase and sulfotransferases (Van der Oost et al., 2003). The third phase included membrane proteins, known as multi-drug resistance or multi-xenobiotic resistance (McFadzen et al., 2000), and acting as a pump involved in the export of xenobiotics out of the cell (Lüdeking and Köhler, 2002).

Flavin-containing monooxygenases catalyse the oxygenation of a wide variety of xenobiotics. They are involved in the four-electron reduction of dioxygen with two electrons derived from reduced nicotinamide cofactor and two electrons derived from substrate (Poulsen and Ziegler, 
1979). FMOs are membrane-bound enzymes found in the smooth endoplasmic reticulum in eucaryotic cells. Several studies reported FMO enzyme activity in molluscs, such as mussels, Mytilus edulis and Mytilus galloprvincialis (Kurelec, 1985; Kurelec and Krca, 1987) or oysters, C. gigas (Schlenk and Buhler, 1989, 1990). To our knowledge, no information are available in database concerning molecular characterisation of FMO in mollusc species.

Monoamine oxidases exist into two forms in mammalian tissues, MAO A and B, differing in their substrate specificity and inhibitor sensitivity (Strolin benedetti, 2001). These enzyme are essentially mitochondrial, although some MAO activity has been reported in micosomes (Wouters, 1998). MAO A enzyme activity has been reported in several tissues and central nervous system of squid in relation with hydrostatic pressure (Youdim et al., 1986; Antipov et al., 1996), but to our knowledge, no investigations were conducted to characterise MAO genes or to detect MAO mRNA expression or enzyme activity in other mollusc species.

In this paper, we characterised, for the first time, the complete cDNA sequences of two nonP450 phase I enzymes, flavin-containing monooxygenase 2 and monoamine oxidase $\mathrm{A}$, in the marine bivalve C. gigas. The mRNA expression of these two enzymes, and their potential use as biomarkers of contaminant exposure were investigated. We also discussed on the regulatory effect of contaminant on FMO-2 and MAO A mRNA expression. We used RT-PCR to analyse FMO-2 and MAO A mRNA expression in oysters exposed to hydrocarbons and two pesticide treatments. One pesticide exposure designated ADI was to a mixture of 2-chlor-4-ethylamino-6isopropylamino-1,3,5,-triazin (atrazine), 3-(3,4-dichlorphenyl)-1-1-dimethyl-harnstoff (diuron) and 3-(4-isopropylphenyl)-1,1-dimethylharnstoff (isoproturon) and the second was to $\mathrm{N}$ (phosphonomethyl)glycine (glyphosate). 


\section{Materials and methods}

\subsection{Experimental design}

Adult oysters (10-11 cm), Crassostrea gigas, were collected from La Pointe du Château (Brittany, France) in Winter 2001 for the hydrocarbon experiment and in Fall 2002 for the pesticide experiments. After an acclimatisation period of 7 days in aerated $0.22 \mu m$-filtered seawater at constant temperature and salinity $\left(15^{\circ} \mathrm{C}\right.$ and $34 \%$, respectively), oysters were challenged as follows. Groups of 20 oysters were exposed for 3 weeks to a $0.1 \%$ mixture of hydrocarbon consisting of the water-soluble fraction of domestic fuel homogenised for three days in filtered seawater (Snyder et al., 2001). Other groups of 20 oysters were exposed for four weeks to either a mixture of three herbicides (atrazine $2 \mu \mathrm{g} / \mathrm{l}$; diuron $0.5 \mu \mathrm{g} / \mathrm{l}$ and isoproturon $1 \mu \mathrm{g} / \mathrm{l}$, mixture called $\mathrm{ADI}$ ) or to $2 \mu \mathrm{g} / \mathrm{l}$ of glyphosate. Another group of 20 oysters was maintained in seawater, without contaminant, as a control. No mortality was observed in the control or treated oysters.

\subsection{Extraction of total RNA and cDNA synthesis (reverse transcription)}

Total RNA were extracted from digestive gland of control and hydrocarbon exposed oysters after $0,7,15$ and 21 days of exposure and from digestive gland and gills of control and pesticide exposed oysters after $0,7,15,21$ and 30 days of exposure according to the method based on

extraction in guanidium isothiocyanate (Strohman et al., 1977). Ten $\mu \mathrm{g}$ RNA were submitted to reverse transcription using oligo $\mathrm{dT}$ anchor primer (GAC CAC GCG TAT CGA TGT CGA $\left.\mathrm{CT}_{(16)} \mathrm{V}\right)$ and $\mathrm{M}-\mathrm{MLV}$ reverse transcriptase (Promega). 
2.3. Cloning and sequencing of 5' and 3' flanking regions of monoamine oxidase $A$ and flavincontaining monooxygenase 2 cDNA

The procedures for the generation of MAO A and FMO-2 cDNA 5' and 3' untranslated regions (UTR) were carried out according to the commercial 5'/3' rapid amplification of cDNA ends protocol (5'/3' RACE Kit, Roche) using specific primers designed from the sequences obtained in suppressive subtraction hybridisation libraries (Boutet et al., in press) and reported in table I. The 3' UTR of MAO A and FMO-2 were amplified as follows: $200 \mathrm{ng}$ of reverse transcription product plus $2 \mathrm{mM} \mathrm{MgCl}_{2}$ and $10 \mathrm{pmol}$ each of PCR anchor primer (GAC CAC GCG TAT CGA TGT CGA C) and specific sense primer was submitted to amplification using one cycle at $94^{\circ} \mathrm{C}$ for $2 \mathrm{~min}, 58^{\circ} \mathrm{C}$ for $2 \mathrm{~min}, 72^{\circ} \mathrm{C}$ for $1 \mathrm{~min} 30$, then 40 cycles at $94^{\circ} \mathrm{C}$ for $15 \mathrm{~s}, 58^{\circ} \mathrm{C}$ for 30 $\mathrm{s}, 72^{\circ} \mathrm{C}$ for $1 \mathrm{~min}$ and a final step at $72^{\circ} \mathrm{C}$ for $10 \mathrm{~min}$. Amplification of the 5 ' UTR was carried out according to the following procedure: denaturation at $94^{\circ} \mathrm{C}$ for $2 \mathrm{~min}$, then 10 cycles at $94^{\circ} \mathrm{C}$ for 15 $\mathrm{s}, 58^{\circ} \mathrm{C}$ for $30 \mathrm{~s}, 72^{\circ} \mathrm{C}$ for $1 \mathrm{~min}$, then 30 cycles at $94^{\circ} \mathrm{C}$ for $15 \mathrm{~s}, 58^{\circ} \mathrm{C}$ for $30 \mathrm{~s}, 72^{\circ} \mathrm{C}$ for 1 min with an increase of $20 \mathrm{~s}$ per cycle for the elongation time, and a final step at $72^{\circ} \mathrm{C}$ for $15 \mathrm{~min}$ with $2 \mathrm{mM}$ $\mathrm{MgCl}_{2}$ and 10 pmol each of oligo dT anchor primer and specific antisense primer. The resulting cDNA fragments corresponding to the 5' and 3' UTRs were cloned into pGEM-T vector (Promega) and sequenced using a Li-COR IR² (Sciencetech) and Thermo Sequenase Primer Cycle Sequencing Kit (Amersham Bioscience).

\subsection{Monoamine oxidase A and flavin-containing monooxygenase 2 expression study by RT-PCR}

MAO A and FMO-2 mRNA expression was analysed in digestive gland of hydrocarbon exposed oysters and in gills and digestive gland of pesticide exposed oysters. PCR was performed using one cycle consisting of denaturation at $94^{\circ} \mathrm{C}$ for $2 \mathrm{~min}$, hybridisation at $58^{\circ} \mathrm{C}$ for 2 min and elongation at $72^{\circ} \mathrm{C}$ for $1 \mathrm{~min}$, then 35 cycles for FMO-2 and 30 cycles for MAO A at $94^{\circ} \mathrm{C}$ for $30 \mathrm{~s}$, 
$58^{\circ} \mathrm{C}$ for $30 \mathrm{~s}, 72^{\circ} \mathrm{C}$ for $30 \mathrm{~min}$, and a final step at $72^{\circ} \mathrm{C}$ for $7 \mathrm{~min}$ with $200 \mathrm{ng}$ of reverse transcription product for FMO-2 amplification and 100 ng of reverse transcription product for MAO A amplification plus $2 \mathrm{mM} \mathrm{MgCl}_{2}$ and 10 pmoles each primers (Table I). A PCR amplification control (28S ribosomal DNA) was used for all experiments and was amplified as described above with primers sense AAG GGC AGG AAA AGA AAC TAA C and antisense TTT CCC TCT AAG TGG TTT CAC. The resulting PCR products were electrophoresed in a $0.5 \mathrm{X}$ TBE/1.5\% agarose gel, and visualised with U/V light after ethidium bromide coloration. Quantification of band intensities was measured by using Gene Profiler 4.03 Software (Scanalytics Inc.).

\section{Results}

\subsection{Identification of the $c D N A$ encoding $M A O A$ in C. gigas}

The cDNA encoding MAO A from C. gigas is contained an open reading frame of 1566 bp (521 amino acids) with a 88 bp-length 5'UTR and a 258 bp-length 3'UTR (Figure 1). The corresponding amino acid sequence contained the characteristic fixation motif of the flavin-adenine dinucleotide (FAD) cofactor (SGGCY, via the cystein 402) and the cystein 370 involved in MAO A enzyme activity. We also observed multiple ATTTA(G) motifs in the untranslated regions of the MAO A correlated with transcript stability. The cDNA encoding MAO A had one ATTTG motif in the 3' UTR. Moreover, the cDNA contained 3 polyadenylation signals in its 3' UTR. A search for amino acid sequence homologies showed that our sequence displayed an homology of 56\% with mammal sequences and dropped to 18\% with Escherichia coli sequence. 
Figure 1: The nucleotide sequence and predicted amino acid sequence of monoamine oxidase A from C. gigas (GenBank accession number AJ556989). Stop codon is marked by an asterisk and untranslated regions are in lower cases. Polyadenylation signals are underlined and messenger stability determining motif ATTTG is in bold characters. Amino acids involved in the fixation of the cofactor FAD are double underlined and the cysteine essential for MAO A activity is boxed.

cttgaggagagcttgcgacaacggtttgggtgaaggcttaggtgttctagataacgtgtctcgactgtctat

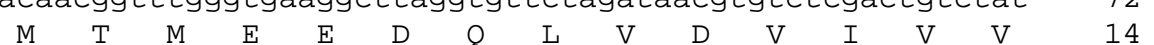
acgaccacatatacac ATG ACT ATG GAG GAA GAT CAG CTG GTG GAT GTT ATT GTT GTA 130 $\begin{array}{lllllllllllllllllll}G & A & G & L & S & G & L & A & A & A & K & L & L & Q & E & T & G & L & 32\end{array}$ GGG GCA GGT CTG AGT GGT TTG GCG GCT GCT AAG CTT TTA CAA GAG ACA GGA CTG 184 $\begin{array}{llllllllllllllllllllll}D & V & L & V & L & E & A & R & D & R & V & G & G & R & T & L & T & E & 50\end{array}$ GAT GTG TTG GTT CTT GAA GCA CGT GAC AGA GTG GGT GGA CGG ACA CTT ACA GAG 238 $\begin{array}{llllllllllllllllllll}H & N & S & H & V & G & Y & V & D & \text { L } & G & G & A & Y & V & G & P & T & 68\end{array}$ CAC AAC TCT CAT GTT GGC TAT GTT GAT TTG GGC GGA GCC TAT GTT GGT CCA ACA 292 \begin{tabular}{lllllllllllllllllll}
$\mathrm{Q}$ & $\mathrm{N}$ & $\mathrm{R}$ & $\mathrm{L}$ & $\mathrm{L}$ & $\mathrm{R}$ & $\mathrm{L}$ & $\mathrm{A}$ & $\mathrm{D}$ & $\mathrm{E}$ & $\mathrm{F}$ & $\mathrm{G}$ & $\mathrm{I}$ & $\mathrm{K}$ & $\mathrm{T}$ & $\mathrm{Y}$ & $\mathrm{F}$ & $\mathrm{T}$ & \\
\hline
\end{tabular} CAG AAC AGA CTC CTG CGT CTG GCG GAT GAA TTC GGG ATT AAA ACT TAT TTT ACC 346 $\begin{array}{llllllllllllllllllllll}N & E & V & E & D & L & V & F & Y & T & K & G & K & S & K & R & Y & H & 104\end{array}$ AAT GAA GTG GAA GAC CTG GTG TTT TAC ACT AAG GGG AAA TCC AAG AGG TAC CAC 400 $\begin{array}{lllllllllllllllllll}G & A & F & S & P & A & S & G & F & F & E & Y & L & D & M & N & N & F & 122\end{array}$ GGC GCC TTT TCC CCA GCA AGT GGG TTT TTC GAG TAT TTG GAC ATG AAT AAT TTT 454 $\begin{array}{lllllllllllllllllll}F & R & L & L & D & K & M & G & E & E & I & P & P & D & A & P & W & R & 140\end{array}$ TTC AGA CTT TTG GAT AAA ATG GGT GAA GAG ATT CCT CCC GAT GCC CCT TGG AGG 508 $\begin{array}{lllllllllllllllllll}A & P & H & A & K & E & W & D & Q & M & T & M & Q & Q & F & L & D & K & 158\end{array}$ GCC CCA CAT GCC AAA GAA TGG GAC CAA ATG ACC ATG CAG CAA TTT CTT GAC AAA 562 $\begin{array}{lllllllllllllllllllll}H & \text { V } & \text { W } & \text { T } & \text { K } & \text { Q } & \text { I } & \text { Y } & \text { R } & \text { F } & \text { C } & \text { K } & \text { T } & \text { F } & \text { V } & \text { S } & \text { V } & \text { N } & 176\end{array}$ CAT GTT TGG ACC AAA CAA ATC TAC CGC TTC TGT AAA ACC TTC GTC AGT GTT AAC 616 $\begin{array}{lllllllllllllllllll}V & T & S & E & P & Y & E & A & S & V & L & W & F & L & W & Y & I & K & 194\end{array}$ GTC ACT TCG GAG CCC TAT GAA GCC TCA GTA CTT TGG TTT CTG TGG TAT ATC AAA 670 $\begin{array}{lllllllllllllllllll}C & C & G & G & Q & K & \text { R } & \text { I } & \text { F } & \text { S } & \text { T } & \text { T } & \text { N } & \text { G } & \text { G } & \text { Q } & \text { E } & \text { R } & 212\end{array}$ TGT TGT GGC GGA CAA AAG CGA ATT TTC TCA ACA ACA AAT GGC GGA CAG GAG AGA 724 $\begin{array}{lllllllllllllllllll}K & F & V & G & G & S & Q & Q & I & S & K & R & I & A & E & K & L & G & 230\end{array}$ AAG TTT GTT GGC GGC TCA CAA CAA ATC AGC AAA AGA ATT GCC GAA AAG CTG GGC 778 $\begin{array}{llllllllllllllllllll}\mathrm{N} & \mathrm{D} & \mathrm{R} & \mathrm{V} & \mathrm{L} & \mathrm{L} & \mathrm{S} & \mathrm{H} & \mathrm{P} & \mathrm{V} & \mathrm{C} & \mathrm{H} & \mathrm{I} & \mathrm{S} & \mathrm{Q} & \mathrm{T} & \mathrm{T} & \mathrm{D} & 248\end{array}$ AAC GAC CGA GTC CTC CTG AGT CAC CCT GTG TGC CAT ATC AGC CAG ACA ACT GAT 832 $\begin{array}{lllllllllllllllllllllll}G & V & T & V & S & V & T & G & G & Q & Q & F & R & A & K & R & V & I & 266\end{array}$ GGA GTG ACG GTG TCC GTT ACT GGT GGA CAA CAA TTC AGG GCT AAA CGT GTC ATT 886 $\begin{array}{lllllllllllllllllll}\mathrm{I} & \mathrm{A} & \mathrm{S} & \mathrm{P} & \mathrm{L} & \mathrm{P} & \mathrm{L} & \mathrm{Q} & \mathrm{N} & \mathrm{K} & \mathrm{I} & \mathrm{T} & \mathrm{Y} & \mathrm{D} & \mathrm{P} & \mathrm{P} & \mathrm{L} & \mathrm{P} & 284\end{array}$ ATT GCC TCT CCG CTA CCT TTA CAA AAT AAG ATC ACA TAT GAC CCT CCC TTG CCA 940 $\begin{array}{lllllllllllllllllll}\mathrm{S} & \mathrm{L} & \mathrm{R} & \mathrm{N} & \mathrm{Q} & \mathrm{L} & \mathrm{I} & \mathrm{Q} & \mathrm{R} & \mathrm{I} & \mathrm{P} & \mathrm{M} & \mathrm{G} & \mathrm{S} & \mathrm{V} & \mathrm{I} & \mathrm{K} & \mathrm{T} & 302\end{array}$ AGT CTT AGG AAT CAA TTA ATA CAA AGA ATC CCG ATG GGT TCC GTC ATC AAA ACA 994 $\begin{array}{lllllllllllllllllll}F & C & Y & Y & K & T & P & F & W & K & E & K & G & Y & C & G & S & T & 320\end{array}$ TTT TGC TAC TAC AAG ACC CCT TTT TGG AAG GAG AAA GGT TAT TGT GGG TCC ACT 1048 $\begin{array}{lllllllllllllllllll}A & I & D & D & D & A & A & I & V & E & F & T & L & D & D & T & K & H & 338\end{array}$ GCA ATA GAC GAT GAT GCA GCC ATA GTT GAA TTC ACA TTG GAT GAT ACG AAG CAT 1102

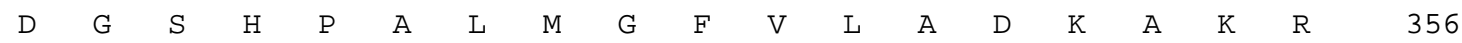
GAC GGA AGT CAC CCA GCG TTA ATG GGA TTT GTT CTT GCA GAT AAG GCA AAA CGT 1156 $\begin{array}{lllllllllllllllllllllll}F & V & S & M & T & P & E & E & K & K & E & S & I & C & R & L & Y & A & 374\end{array}$ TTT GTT TCT ATG ACA CCG GAA GAG AAA AAA GAG AGT ATA TGT CGT CTG TAT GCT 1210 $\begin{array}{lllllllllllllllllll}K & V & F & K & S & D & E & A & L & Y & P & I & H & Y & E & E & K & N & 392\end{array}$ AAA GTC TTC AAA TCA GAC GAA GCG TTA TAT CCA ATA CAC TAT GAA GAG AAG AAC 1264 $\begin{array}{lllllllllllllllllll}W & L & G & E & Q & W & S & G & G & C & Y & T & A & M & M & P & P & G & 410\end{array}$

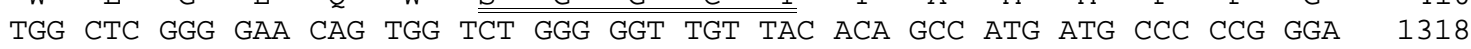
$\begin{array}{llllllllllllllllllll}\mathrm{F} & \mathrm{L} & \mathrm{T} & \mathrm{N} & \mathrm{F} & \mathrm{G} & \mathrm{E} & \mathrm{E} & \mathrm{I} & \mathrm{R} & \mathrm{R} & \mathrm{P} & \mathrm{V} & \mathrm{G} & \mathrm{N} & \mathrm{L} & \mathrm{Y} & \mathrm{F} & 428\end{array}$ TTC CTG ACC AAC TTC GGC GAA GAA ATA AGA AGA CCC GTT GGT AAT TTA TAT TTC 1372 $\begin{array}{lllllllllllllllllll}A & G & T & E & T & A & T & Q & W & S & G & Y & M & E & G & A & V & Q & 446\end{array}$ GCG GGG ACA GAG ACA GCC ACC CAG TGG TCG GGG TAC ATG GAA GGG GCG GTC CAG 1426 $\begin{array}{llllllllllllllllllll}A & G & E & R & A & A & R & E & I & L & F & D & M & K & K & I & P & K & 464\end{array}$ GCC GGG GAG AGG GCG GCC AGA GAG ATT TTA TTT GAC ATG AAA AAA ATT CCC AAA 1480 $\begin{array}{lllllllllllllllllll}Y & E & I & W & Q & D & E & E & E & N & T & L & V & R & A & R & P & F & 482\end{array}$ TAT GAA ATT TGG CAA GAC GAA GAA GAG AAT ACT CTT GTG AGA GCC CGT CCG TTT 1534 $\begin{array}{lllllllllllllllllll}E & S & T & F & W & E & R & N & L & P & S & V & G & G & F & L & K & C & 500\end{array}$ GAG AGC ACG TTT TGG GAG AGA AAC CTA CCG TCA GTT GGG GGG TTT CTG AAG TGT 1588 $\begin{array}{lllllllllllllllllll}V & S & V & T & T & A & L & A & V & G & S & A & G & L & C & L & Y & W & 518\end{array}$ GTA TCC GTT ACA ACA GCT TTA GCA GTC GGA TCG GCG GGA CTG TGT CTT TAT TGG 1642 W $\mathrm{K} \quad \mathrm{R}$ * $\quad 522$ TGG AAA CGT TAA catgctaattcaattaaaaaatgaccagattcaaaataacctagggttttatctt 1709 aaaagatcaaattaaataaggctcagtaaatcaaaaaataaatcaaacattcaagtatatttgtttgcatct 1781

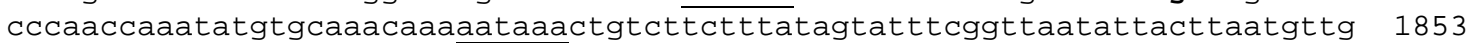
ctggattctttgacacttgaaataaagatattgaataaaaaaaaaaaaaaaaaaa

1853 
The cDNA encoding FMO-2 from C. gigas is contained an open reading frame of 1356 bp (451 amino acids) with a 24 bp-length 5'UTR and a 36 bp-length 3'UTR (Figure 2). The corresponding amino acid sequence contained the characteristic fixation motif of the FAD cofactor (GAGPAG). The FMO-2 cDNA sequence showed a short 3'UTR and the polyadenylation signal overlapped the third base of the last codon and the stop codon (AGA TAA A). The amino acid sequence homology with mammal sequences is $24 \%$.

Figure 2: The nucleotide sequence and predicted amino acid sequence of flavin-containing monooxygenase 2 from C. gigas (GenBank accession number AJ585074). Stop codon is marked by an asterisk and untranslated regions are in lower cases. Polyadenylation signal is underlined. Amino acids involved in the fixation of the cofactor FAD are boxed.

\begin{tabular}{|c|c|c|c|c|c|c|c|c|c|c|c|c|c|c|c|c|c|c|}
\hline & & & & & & $\mathrm{N}$ & & & $\mathbf{T}$ & $\mathbf{T}$ & T & $\mathbf{R}$ & & $\mathbf{R}$ & V & A & V & 18 \\
\hline ATAC & CACG] & ГACAC & GTGA & GCAG & TAGAC & G ATC & G TCG & $\mathbf{G} \mathbf{G G C}$ & $\mathbf{G} \mathrm{AC}$ & $A \quad A C$ & T GGC & C $A G A$ & A CAC & G AGG & G GTR & R GCG & ; GTT & 60 \\
\hline I & $\mathbf{G}$ & $A$ & G & $\mathbf{P}$ & $A$ & $\mathrm{G}$ & $\mathbf{L}$ & C & C & V & K & H & L & A & A & K & $\mathbf{P}$ & 30 \\
\hline ATC & GGG & GCC & GGA & CCG & GCG & $\mathbf{G G G}$ & CTG & TGC & TGT & GTC & AAG & CAT & TTG & GCT & GCG & AAA & ccc & 114 \\
\hline E & $\mathbf{L}$ & $\mathbf{F}$ & $E$ & $\mathbf{P}$ & V & A & $\mathbf{r}$ & E & $\mathbf{R}$ & $\mathbf{N}$ & $\mathbf{F}$ & W & $\mathbf{P}$ & G & G & I & W & 48 \\
\hline GAG & СтC & TTT & GAA & CCT & GTT & GCC & TTT & GAG & CGT & AAC & ;TT & TGG & CCG & GGC & GGG & ATT & TGG & 168 \\
\hline $\mathbf{N}$ & $\mathbf{Y}$ & $\mathbf{T}$ & D & $\mathbf{Q}$ & $\mathbf{T}$ & $\mathbf{R}$ & K & D & A & $\mathbf{F}$ & G & L & $\mathbf{P}$ & V & H & $s$ & A & 66 \\
\hline AAC & TAC & АCT & GAC & CAG & ACC & CGG & AAA & GAC & GCA & TTC & GGA & CTT & CCG & GTA & CAC & TCT & GCA & 222 \\
\hline L & $\mathrm{Y}$ & $\mathbf{N}$ & K & L & $\mathbf{K}$ & I & $\mathbf{N}$ & V & $\mathbf{P}$ & K & E & L & Q & E & $F$ & $\mathbf{P}$ & s & 84 \\
\hline CTG & TAT & AAT & AAG & CTG & AAG & ATA & AAT & GTT & CCA & AAA & GAA & CTA & CAG & GAA & TTT & CCA & AGT & 276 \\
\hline $\mathbf{F}$ & $\mathbf{P}$ & $Y$ & $\mathbf{P}$ & K & E & W & K & $\mathbf{T}$ & $S$ & $\mathbf{Y}$ & I & $\mathbf{T}$ & $\mathbf{R}$ & Q & Q & C & W & 102 \\
\hline TTC & CCC & TAC & CCA & AAG & GAA & TGG & AAG & ACG & TCC & TAC & ATC & ACG & CGG & CAG & CAG & TGC & TGG & 330 \\
\hline $\mathrm{E}$ & $\mathrm{Y}$ & $\mathbf{L}$ & $\mathbf{N}$ & M & $\mathbf{F}$ & $\mathbf{T}$ & D & H & $\mathbf{F}$ & D & I & $\mathbf{R}$ & K & $\mathbf{Y}$ & I & $\mathbf{R}$ & $\mathbf{F}$ & 120 \\
\hline GAA & TAC & CTC & AAC & ATG & TTC & ACA & GAC & CAT & TTC & GAC & ATC & AGG & AAG & TAT & ATC & CGG & TTT & 384 \\
\hline H & $\mathbf{S}$ & $\mathbf{F}$ & $\mathbf{v}$ & $\mathbf{R}$ & $\mathbf{N}$ & V & K & $\mathbf{P}$ & $\mathbf{L}$ & K & E & M & $\mathbf{N}$ & E & $\mathbf{N}$ & G & K & 138 \\
\hline CAT & TCA & TTT & GTT & CGA & AAC & GTG & AAG & CCA & TTG & AAG & GAG & ATG & AAC & GAA & AAC & GGC & AAA & 438 \\
\hline $\mathbf{P}$ & K & W & L & V & $\mathbf{T}$ & $\mathbf{F}$ & $S$ & $\mathbf{P}$ & V & $\mathbf{T}$ & $\mathbf{R}$ & M & $S$ & E & V & $\mathbf{N}$ & $\mathbf{T}$ & 156 \\
\hline CCA & AAA & TGG & CTG & GTG & ACC & TTT & TCA & ccc & GTG & ACC & CGA & ATG & TCA & GAG & GTC & AAC & ACA & 492 \\
\hline E & v & $\mathbf{F}$ & D & A & V & $\mathbf{L}$ & V & $\mathbf{S}$ & $\mathbf{N}$ & G & H & D & F & $\mathbf{N}$ & D & $\mathbf{Y}$ & $\mathbf{T}$ & 174 \\
\hline GAA & GTG & TTC & GAC & GCC & GTA & СTT & GTG & AGC & AAC & GGC & : CAC & GAC & TTC & AAC & GAC & TAC & ACG & 546 \\
\hline $\mathbf{P}$ & $\mathbf{N}$ & I & $P$ & $\mathbf{G}$ & L & E & L & $\mathbf{F}$ & E & G & R & A & I & H & $\mathbf{S}$ & K & E & 92 \\
\hline CcC & AAC & ATT & CcC & GGC & CTG & GAG & CTG & TTT & GAG & GGA & CGA & GCT & ATC & САT & AGC & AAG & GAG & 600 \\
\hline $\mathbf{F}$ & $\mathbf{R}$ & $\mathbf{Y}$ & E & E & H & $\mathbf{F}$ & D & G & L & $\mathbf{R}$ & V & A & I & L & G & C & H & 210 \\
\hline TTC & CGG & TAC & GAG & GAA & CAC & TTT & GAC & GGA & СTT & CGT & GTG & GCG & ATC & CTG & GGC & TGC & CAC & 654 \\
\hline $\mathbf{Y}$ & $S$ & G & E & D & I & $\mathrm{S}$ & $\mathbf{T}$ & H & V & A & K & $\mathbf{F}$ & A & K & K & v & $\mathrm{Y}$ & 28 \\
\hline TAT & TCC & GGA & GAA & GAC & ATC & TCT & ACG & CAT & GTC & GCT & AAG & TTT & GCT & AAG & AAG & GTA & TAC & 708 \\
\hline A & C & H & $\mathbf{R}$ & $\mathbf{R}$ & $\mathbf{N}$ & $\mathbf{P}$ & K & E & $\mathbf{F}$ & $\mathbf{P}$ & P & $\mathbf{s}$ & $\mathbf{F}$ & $\mathbf{P}$ & K & E & I & 246 \\
\hline GCC & TGT & CAT & CGA & AGA & AAT & CCA & AAG & GAA & TTT & CCA & A CCG & TCT & TTC & CCG & AAG & GAA & ATC & 762 \\
\hline $\mathrm{E}$ & $\mathbf{Q}$ & $\mathbf{R}$ & $\mathbf{P}$ & $\mathbf{P}$ & $\mathbf{F}$ & A & $\mathbf{R}$ & M & $\mathbf{T}$ & $\mathbf{R}$ & D & $\mathbf{S}$ & V & V & $\mathbf{F}$ & $\mathbf{P}$ & D & 264 \\
\hline GAA & CAA & CGA & CCA & CCG & TTC & GCC & CGC & ATG & ACC & AGA & A GAT & TCA & GTG & GTT & TTC & CCG & GAC & 816 \\
\hline G & G & $S$ & E & K & V & D & A & V & I & $\mathbf{F}$ & C & $\mathbf{T}$ & G & $\mathrm{Y}$ & $\mathbf{R}$ & $\mathbf{F}$ & $S$ & 82 \\
\hline GGA & GGT & TCC & GAG & AAG & GTG & GAC & GCC & GTT & ATA & TTC & TGC & ACC & GGA & TAT & CGC & TTC & TCT & 870 \\
\hline $\mathbf{Y}$ & $\mathbf{P}$ & $\mathbf{F}$ & $\mathbf{L}$ & K & D & D & V & I & $\mathbf{T}$ & I & K & D & E & $\mathbf{R}$ & I & E & $\mathbf{P}$ & 300 \\
\hline TAT & CCA & TTT & TTA & AAG & GAT & GAC & GTC & ATC & ACA & ATC & AAG & GAT & GAG & AGG & ATA & GAG & CcC & 924 \\
\hline I & $Y$ & K & H & M & V & H & I & E & $\mathrm{Y}$ & $\mathbf{N}$ & N & L & I & $\mathbf{F}$ & V & G & I & 318 \\
\hline АTT & TAC & AAA & CAC & ATG & GTG & CAC & ATT & GAG & TAC & AAC & AAC & TTG & ATA & TTT & GTG & GGA & ATT & 978 \\
\hline $\mathbf{P}$ & $\mathbf{R}$ & $\mathbf{Q}$ & W & $\mathrm{s}$ & $\mathrm{Y}$ & $\mathbf{F}$ & $\mathbf{P}$ & H & $\mathrm{Y}$ & H & E & M & A & K & L & A & A & 336 \\
\hline CCC & CGA & CAA & TGG & TCG & TAC & TTT & CCC & CAC & TAT & CAC & GAA & ATG & GCA & AAA & СTT & GCT & GCG & 1032 \\
\hline $\mathbf{L}$ & I & L & A & E & D & V & K & L & $\mathbf{P}$ & S & K & E & I & M & L & A & D & 354 \\
\hline TTA & ATT & TTG & GCG & GAA & GAC & GTC & AAA & CTG & CCG & TCC & AAA & GAG & ATC & ATG & CTG & GCG & GAC & 1086 \\
\hline $\mathbf{S}$ & E & A & D & $\mathbf{F}$ & Q & $\mathbf{S}$ & $\mathbf{R}$ & $\mathbf{L}$ & K & E & G & K & $\mathbf{P}$ & $\mathbf{P}$ & $\mathbf{S}$ & $\mathbf{F}$ & A & 372 \\
\hline AGC & GAG & GCC & GAT & TTC & CAG & TCG & CGT & TTG & AAA & GAA & A GGA & AAG & CCG & ССТ & TCG & TTT & GCC & 1140 \\
\hline H & $\mathbf{Y}$ & M & G & D & I & D & $\mathbf{R}$ & Q & $\mathbf{F}$ & $\mathbf{R}$ & $Y$ & $\mathbf{N}$ & E & D & L & A & K & 390 \\
\hline САT & TAC & ATG & GGG & GAC & ATC & GAC & CGT & CAG & TTC & CGG & G TAT & AAC & GAG & GAC & CTG & GCA & AAG & 1194 \\
\hline M & $\mathbf{G}$ & G & $\mathbf{F}$ & D & $\mathbf{P}$ & $\mathbf{L}$ & $\mathbf{P}$ & $\mathbf{P}$ & V & L & E & M & M & W & D & D & V & 408 \\
\hline ATG & GGC & GGG & TTT & GAC & CCA & СTT & CCG & ССТ & GTC & CTG & G GAG & ATG & ATG & TGG & GAT & GAC & GTC & 1248 \\
\hline M & D & $\mathrm{E}$ & $\mathbf{R}$ & $\mathbf{Y}$ & M & $\mathbf{N}$ & $\mathbf{L}$ & $\mathbf{P}$ & $\mathbf{N}$ & C & $\mathbf{N}$ & $\mathbf{T}$ & $\mathbf{F}$ & D & $\mathbf{Y}$ & E & I & 426 \\
\hline ATG & GAT & GAG & AGG & TAC & ATG & AAC & СTC & CCG & AAC & TGT & I AAC & ACT & TTT & GAC & TAT & GAG & ATC & 1302 \\
\hline $\mathbf{T}$ & G & $\mathbf{P}$ & $Q$ & $\mathbf{S}$ & $Y$ & $\mathbf{R}$ & C & L & $\mathbf{N}$ & $\mathbf{P}$ & E & G & I & K & $\mathbf{T}$ & $\mathbf{R}$ & L & 444 \\
\hline ACA & GGA & CCA & CAG & TCC & TAC & CGC & TGT & CTG & AAC & CCC & GAG & GGA & ATC & AAA & ACC & AGG & TTG & 1356 \\
\hline C & K & A & E & $\mathbf{N}$ & v & K & $\mathbf{R}$ & * & & & & & & & & & & 453 \\
\hline TGT & AAG & GCT & GAA & AAT & GTG & AAG & AGA & TAA & AA & & TG & ГCC & & & AA & & & 1416 \\
\hline
\end{tabular}




\subsection{MAO A expression analysis using RT-PCR}

The results of RT-PCR showed a strong differential MAO A expression between tissues and treatments. Analysis of MAO A expression in the digestive gland of oysters exposed to hydrocarbons displayed an induction of MAO A mRNA synthesis after 21 days of exposure (1.4fold compared with control) (Figure 3).

Figure 3: Expression of MAO A in digestive gland of oysters exposed to hydrocarbons by RT-PCR ( $n=3$ for each day). Lane $1: 100$ bp marker; Lane 2: control oysters; Lane 3: oysters exposed for 7 days; Lane 4: oysters exposed for 15 days; Lane 5: oysters exposed for 21 days.

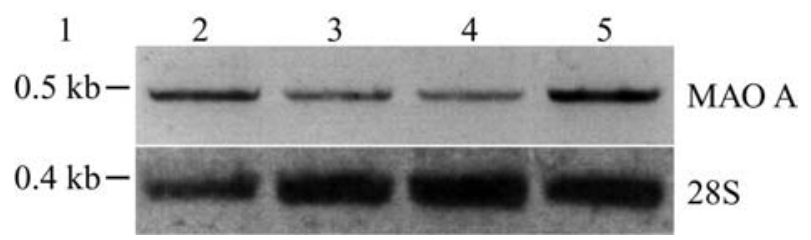

Regarding pesticide exposure results, we observed that both treatments inhibit MAO A mRNA synthesis in gills of oysters (Figures 4A and B). Then, we showed that an exposure to ADI entailed an inhibition of MAO A at 7 days, followed by an induction after 15 days, reaching a maximum value at 30 days in the digestive gland (1.8-fold compared with control) (Figure 4A). The expression of MAO A in digestive gland of glyphosate exposed oysters showed a strong induction at 7 days which is maintained until the end of the experiment (2.5-fold compared with control)

(Figure 4B).

Figure 4: Expression of MAO A in digestive gland (lanes 2 to 6) and gills (lanes 7 to 11) of oysters exposed to ADI (A) or glyphosate (B) by RT-PCR ( $n=3$ for each day). Lane 1: 100 bp marker; Lanes 2 and 7: control oysters; Lanes 3 and 8: oysters exposed for 7 days; Lanes 4 and 9: oysters exposed for 15 days; Lanes 5 and 10: oysters exposed for 21 days; Lanes 6 and 11: oysters exposed for 30 days.

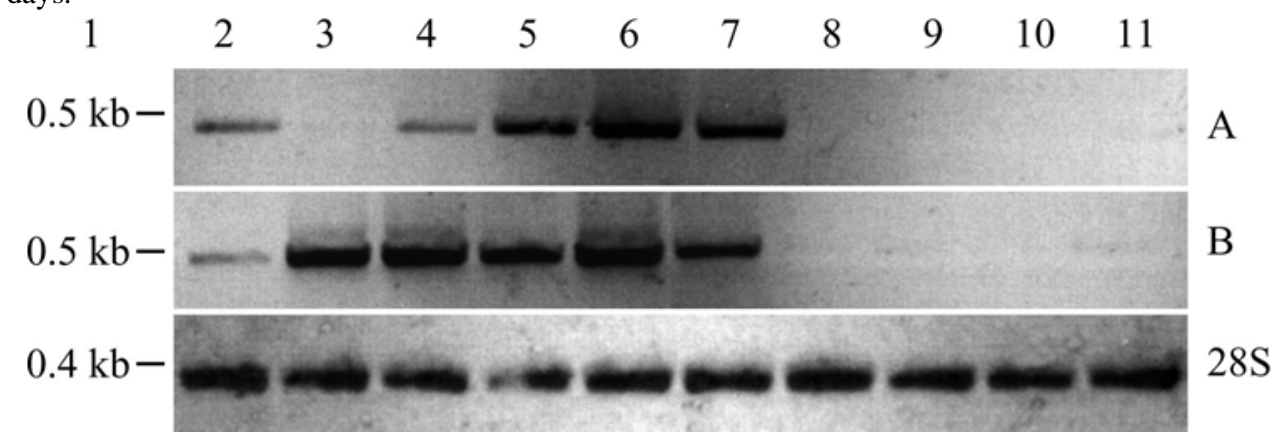




\subsection{FMO-2 expression analysis using RT-PCR}

The analysis of FMO-2 expression showed differences between tissues and treatments. An exposure to hydrocarbons entailed an induction at 7 days in digestive gland of oysters (2.2-fold compared with control), before dropping to the level observed in control oysters (Figure 5).

Regarding pesticide exposure results, we observed that FMO-2 is not expressed in gills of pesticide-exposed oysters (Figures 6A and B). We observed that both ADI and glyphosate exposure entailed an induction after 30 days in digestive gland (3.5- and 3-fold compared with control, respectively) (Figure 6A and B).

Figure 5: Expression of FMO-2 in digestive gland of oysters exposed to hydrocarbons by RT-PCR ( $n=3$ for each day). Lane 1: 100 bp marker; Lane 2: control oysters; Lane 3: oysters exposed for 7 days; Lane 4: oysters exposed for 15 days; Lane 5: oysters exposed for 21 days

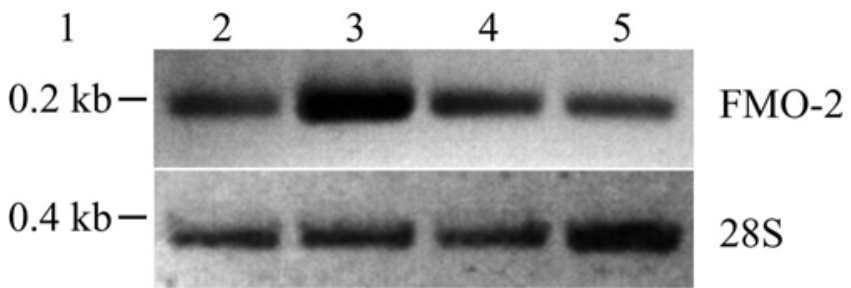

Figure 6: Expression of FMO-2 in digestive gland (lanes 2 to 6 ) and gills (lanes 7 to 11) of oysters exposed to ADI (A) or glyphosate (B) by RT-PCR ( $n=3$ for each day). Lane 1: 100 bp marker; Lanes 2 and 7: control oysters; Lanes 3 and 8: oysters exposed for 7 days; Lanes 4 and 9: oysters exposed for 15 days; Lanes 5 and 10: oysters exposed for 21 days; Lanes 6 and 11: oysters exposed for 30 days.

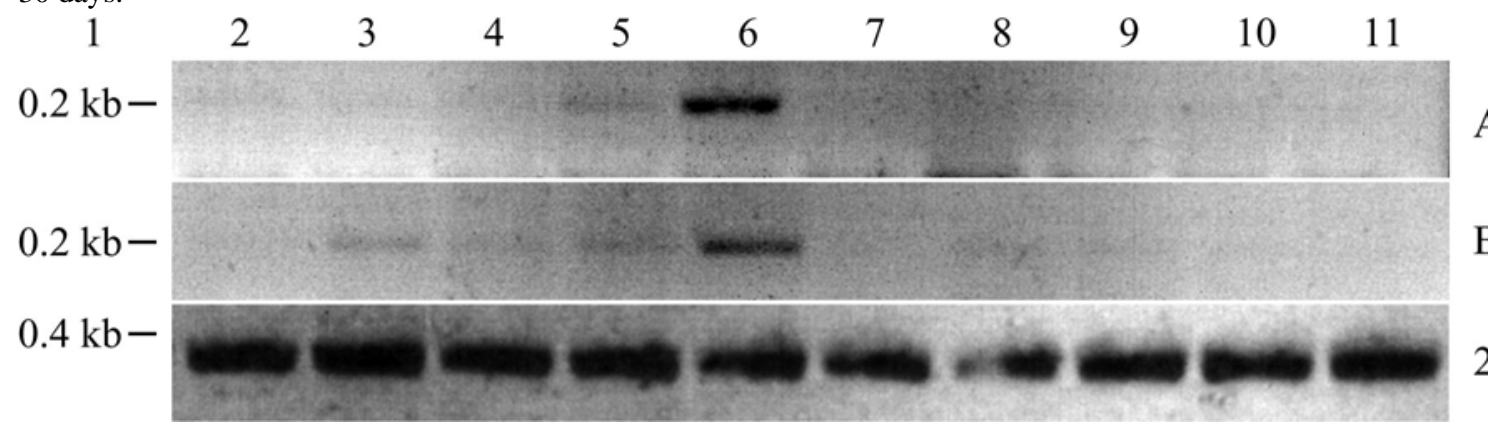

A

B

$28 \mathrm{~S}$

\section{Discussion}

In the present paper, we reported, for the first time, the complete sequences of two non-P450 enzymes involved in xenobiotic biotransformation, monoamine oxidase A and flavin-containing 
monooxygenase 2, from the Pacific oyster $C$. gigas. We previously obtained two partial sequences in a digestive gland SSH library from oysters exposed to hydrocarbons (Boutet et al., in press). Specific primers were then designed from these sequences to amplified the complete MAO A and FMO-2 cDNAs. The two sequences reported here presented the characteristic FAD-fixation motifs of the two enzyme families studied: SGGCY (via the cyteine 402) for the MAO A (Strolin Benedetti, 2001) and GAGPAG for the FMO-2. We also observed the cystein 370 involved in MAO A enzyme activity (Wouters, 1998). The FAD-fixation motif of the FMO-2 is present in other species sequences as GAGVSG in monkey (Yueh et al., 1997), rabbit (Lawton et al., 1990) and guinea pig (Nikbakht et al., 1992). A fixation motif for $\beta$-nicotinamide adenine dinucleotide phosphate (NADP) was reported in monkey and human FMO (GMGNSG) (Phillips et al., 1995; Yueh et al., 1997) and rabbit and guinea pig FMO (GIGNSA) (Lawton et al., 1990; Nikbakht et al., 1992), but this motif was not present in our sequence. The low degree of homology between oyster and mammal sequence certainly explain the lack of similar NADP fixation motif. This motif is probably present in oyster sequence, involving other amino acids that those described above. The cDNA sequence encoding FMO-2 in C. gigas presented another interesting feature consisting in a short 3'UTR and a polyadenylation signal which overlapped the third base of the last codon and the stop codon (AG $\underline{\text { TAA A }}$. This particularity was previously observed in sequence encoding AMP desaminase in rat (Sabina et al., 1987). The MAO A cDNA sequence presented one ATTTG motif in its 3'UTR. These motifs are known to be correlated with transcript stability (Shaw and Kamen, 1986) and have been observed in other cDNAs, such as the sequence encoding glutamine synthetase in the sea urchin Paracentrotus lividus (Fucci et al., 1995), in rat aspartate aminotransferase (AAT) (Pavé-Preux et al., 1988) and in C. gigas pi class GST (GenBank accession number AJ557140). Another interesting 3'UTR feature seen was three polyadenylation signals in the MAO A cDNA sequence. Multiple polyadenylation sites have already been observed for other genes (Leff et al., 1986). Caizzi et al. (1990) and Smartt et al. (1998 and 2001) suggest that the multiple polyadenylation sites indicate the presence of multiple transcripts encoding one protein. Pavé-Preux 
et al. (1988) found that a single AAT sequence containing two polyadenylation signals encoded two different mRNAs in rat. They postulated that the two mRNAs resulted from the differential use of these signals during the maturation of pre-mRNA. More analysis will be necessary to determine how many different mRNAs are encoding by the C. gigas MAO A cDNA sequence.

Analysis of FMO-2 expression by RT-PCR in xenobiotic-exposed oysters, showed an increase of mRNA synthesis in the digestive gland of all treated oysters, whereas an absence of FMO-2 expression was observed in gills. Conversely, Schlenk and Buhler (1989) detected FMO enzyme activity in gills of $C$. gigas. Other studies showed that FMO enzyme activity was normally greater in the digestive gland/hepatopancreas/liver than in other organ (Ziegler, 1988, 1993). It is also assumed that gills contained a limited yield of microsomes (Schlenk and Buhler, 1989) and it could explain the lack of FMO mRNA expression in gills of pesticide-exposed oysters in our analysis. More, FMO activity was detected in digestive gland and visceral mass of mussels and oysters (Kurelec, 1985; Kurelec and Krca, 1987; Livingstone et al., 1990; Schlenk and Buhler, 1990). Detection of FMO activity and mRNA expression in the digestive gland of bivalves suggests that this system plays a key role in xenobiotic detoxification.

The second Phase I enzyme studied here is the monoamine oxidase A. In the present report, we investigated mRNA expression by RT-PCR in hydrocarbon and pesticide exposed oysters. We observed a strong induction of MAO A in digestive gland of all treated oysters, while pesticide exposure entailed an inhibition of MAO A expression in gills. We also shown a similar inhibitory effect of pesticides on mRNA synthesis of two glutathione S-transferases (GSTs) in C. gigas (data not shown). Nevertheless, previous investigations on MAOs showed that these enzymes are constitutive and presented no known external regulation of its expression (Ramsay, 1998). In our study, we confirmed the constitutive status of MAO A in oysters, because mRNA synthesis was detected in both tissues of control oysters. More, we previously obtained MAO A partial sequence in an up-regulated SSH library (Boutet et al., in press), that it could have not been possible if MAO A was only constitutively expressed. Our study clearly demonstrate that MAO A mRNA expression 
is regulated by xenobiotic exposure in oysters, either characterised by an induction in digestive gland or by an inhibition in gills.

In this study, we characterised for the first time, two complete cDNAs encoding non-P450 enzymes involved in xenobiotic detoxification, monoamine oxidase A and flavin containing monooxygenase 2, in the Pacific oyster $C$. gigas. The two amino acid sequences contained the characteristic motifs of the enzyme families and some interesting features described in other sequences. Using these two sequences, we investigated mRNA expression in tissues of pesticideand hydrocarbon-exposed oysters under experimental conditions. These two enzymes are strongly time- and tissue-regulated by all xenobiotics tested here. More, according to the present study and work on GSTs in C. gigas, we could hypothesise that pesticides have an inhibitory effect on mRNA synthesis of several biotransformation enzymes in gills. The analysis of MAO A and FMO-2 mRNA level could be use as a biomarker of pesticide or hydrocarbon exposure in oysters from contaminated estuaries.

\section{Acknowledgements}

This research program was supported by the Région Bretagne, the Conseil Général du Finistère and the interregional program MOREST (Summer Mortality of juvenile oyster Crassostrea gigas). The authors are grateful to Brenda J. Landau for English corrections and to Monique Briand for editing the figures.

\section{References}

Antipov, A.D., Kostkin, V.B., Rozengart, E.V., Epshtein, L.M. 1996. A comparative analysis of monoamine oxidase activity in the tissues of the deep-sea squid (Berryteuthis magister) and the mouse under normal and elevated atmospheric pressure. Zh. Evol. Biokhim. Fiziol. 32, 233-240. 
Beedham, C. 1997. The role of non-P450 enzymes in drug oxidation. Pharm. World. Sci. 19, 255263.

Boutet, I., Tanguy, A., Auffret, M., Riso, R., Moraga, D. 2002. Immunochemical quantification of metallothioneins in marine mollusks: characterization of a metal exposure bioindicator. Environ. Toxicol. Chem. 21, 1009-1014.

Boutet, I., Tanguy, A., Rousseau, S., Auffret, M., Moraga, D. 2003. Molecular identification and expression of Heat Shock Cognate (hsc70) and Heat Shock Protein (hsp70) genes in the Pacific oyster Crassostrea gigas. Cell Stress Chap. 8, 76-85.

Boutet, I., Tanguy, A., Moraga, D. Response of the Pacific oyster Crassostrea gigas to hydrocarbon contamination under experimental conditions. Gene. In press.

Caizzi, R., Bozzetti, M.P., Caggese, C., Ritossa, F. 1990. Homologous nuclear genes encode cytoplasmic and mitochondrial glutamine synthetase in Drosophila melanogaster. J. Mol. Biol. 212, $17-26$.

Fucci, L., Piscopo, A., Aniello, F., Branno, M., Di Gregorio, A., Calogero, R., Geraci, G. 1995. Cloning and characterization of a developmentally regulated sea urchin cDNA encoding glutamine synthetase. Gene. 152, 205-208.

Kurelec, B. 1985. Exclusive activation of aromatic amines in the marine mussel Mytilus edulis by FAD-containing monooxygenase. Biochem. Biophys. Res. Commun. 127, 773-778.

Kurelec, B., Krca, S. 1987. Metabolic activation of 2-aminofluorene, 2-acetylaminofluorene and Nhydroxy-acetylaminofluorene to bacterial mutagens with mussel (Mytilus galloprovincialis) and carp (Cyprinus carpio) subcellular preparations. Comp. Biochem. Physiol. 88 C, 171-177.

Lawton, M.P., Gasser, R., Tynes, R.E., Hodgson, E., Philpot, R.M. 1990. The flavin-containing monooxygenase enzymes expressed in rabbit liver and lung are products of related but distinctly different genes. J. Biol. Chem. 265, 5855-5861.

Leff, S.E., Rosenfeld, M.G., Evans, R.M. 1986. Complex transcriptional units: diversity in gene expression by alternative RNA processing. Annu. Rev. Biochem. 55, 1091-1117. 
Livingstone, D.R., Arnold, R., Chipman, K., Kirchin, M.A., Marsh, J. 1990. The mixed function oxygenase system in molluscs: metabolism, responses to xenobiotics and toxicity. Oceanis. 16, 331-347.

Lüdeking, A., Köhler, A. 2002. Identification of six mRNA sequences of genes related to multixenobiotic resistance (MXR) and biotransformation in Mytilus edulis. Mar. Ecol. Prog. Ser. 238, 115-124.

McFadzen, I., Eufemia, N., Heath, C., Epel, D., Moore, M., Lowe, D. 2000. Multidrug resistance in the embryos and larvae of the mussel Mytilus edulis. Mar. Environ. Res. 50, 319-323.

Michel, X.R., Cassand, P.M., Ribera, D.G., Narbonne, J.F. 1992. Metabolism and mutagenic activation of benzo[a]pyrene by subcellular fractions from mussel (Mytilus galloprovincialis) digestive gland and sea bass (Dicenthrarcus labrax) liver. Comp. Biochem. Physiol. 103 C, 4351.

Nikbakht, K.N., Lawton, M.P., Philpot, R.M. 1992. Guinea pig or rabbit lung flavin-containing monooxygenases with distinct mobilities in SDS-PAGE are allelic variants that differ at only two positions. Pharmacogenetics. 2, 207-216.

Pavé-Preux, M., Ferry, N., Bouguet, J., Hanoune, J., Barouki, R. 1988. Nucleotide sequence and glucocorticoid regulation of the mRNAs for the isoenzymes of rat aspartate aminotransferase. J. Biol. Chem. 263, 17459-17466.

Phillips, I.R., Dolphin, C.T., Clair, P., Hadley, M.R., Hutt, A.J., McCombie, R.R., Smith, R.L., Shephard, E.A. 1995. The molecular biology of the flavin-containing monooxygenases of man. Chem. Biol. Interact. 96, 17-32.

Poulsen, L.L., Ziegler, D.M. 1979. The liver microsomal FAD-containing monooxygenase. Spectral characterization and kinetic studies. J. Biol. Chem. 254, 6449-6455.

Ramsay, R.R. 1998. Substrate regulation of monoamine oxidases. J. Neural. Transm. 52, 139-147. 
Sabina, R.L., Marquetant, R., Desai, N.M., Kaletha, K., Holmes, E.W. 1987. Cloning and sequence of rat myoadenylate deaminase cDNA. Evidence for tissue-specific and developmental regulation. J. Biol. Chem. 262, 12397-12400.

Schlenk, D. 1998. Occurence of flavin-containing monooxygenases in non-mammalian eukaryotic organisms. Comp. Biochem. Physiol. 121 C, 185-195.

Schlenk, D., Buhler, D.R. 1989. Xenobiotic biotransformation in the Pacific oyster Crassostrea gigas. Comp. Biochem. Physiol. 94 C, 476-480.

Schlenk, D., Buhler, D.R. 1990. The in vitro biotransformation of 2-aminofluorene in the visceral mass of the Pacific oyster, Crassostrea gigas. Xenobiotica. 20, 563-72.

Shaw, G., Kamen, R.A. 1986. Conserved AU sequence from the 3' untranslated region of GM-CSF mRNA mediates selective mRNA degradation. Cell. 46, 659-667.

Smartt, C.T., Chiles, J., Lowenberger, C., Christensen, B.M. 1998. Biochemical analysis of a blood meal-induced Aedes aegypti glutamine synthetase gene. Insect. Biochem. Mol. Biol. 28, 935945.

Smartt, C.T., Kiley, L.M., Hillyer, J.F., Dasgupta, R., Christensen, B.M. 2001. Aedes aegypti glutamine synthetase: expression and gene structure. Gene. 274, 35-45.

Snyder, M.J., Girvetz, E., Mulder, E.P. 2001. Induction of marine mollusc stress proteins by chemical or physical stress. Arch. Environ. Contam. Toxicol. 41, 22-29.

Strolin Benedetti, M. 2001. Biotransformation of xenobiotics by amine oxidases. Fundam. Clin. Pharmacol. 15, 75-84.

Tanguy, A., Mura, C., Moraga, D. 2001. Cloning of a metallothionein gene and characterization of two other cDNA sequences of the Pacific oyster Crassostrea gigas. Aquat. Toxicol. 55, 35-47.

Tanguy, A., Moraga, D. 2001. Cloning and characterization of a gene coding for a novel metallothionein in the Pacific oyster Crassostrea gigas (CgMT2): a case of adaptive response to metal-induced stress? Gene. 273, 123-130. 
Tanguy, A., Boutet, I., Bonhomme, F., Boudry, P., Moraga, D. 2002. Polymorphism of metallothionein genes in the Pacific oyster Crassostrea gigas as a biomarker of response to metal exposure. Biomarkers. 7, 439-450.

Van der Oost, R., Beyer, J., Vermeulen, N.P.E. 2003. Fish bioaccumulation and biomarkers in environmental risk assessment: a review. Environ. Toxicol. Pharmacol. 13, 57-149.

Wouters, J. 1998. Structural aspects of monoamine oxidase and its reversible inhibition. Curr. Med. Chem. 5, 137-162.

Youdim, M.B., Feldman, S.C., Pappas, G.D., Pollard, H.B. 1986. Serotonin metabolism and the nature of monoamine oxidase in squid central nervous system. Brain Res. 381, 300-304.

Yueh, M.F., Krueger, S.K., Williams, D.E. 1997. Pulmonary flavin-containing monooxygenase (FMO) in rhesus macaque: expression of FMO2 protein, mRNA and analysis of the cDNA. Biochim. Biophys. Acta. 1350, 267-271.

Ziegler, D.M. 1988. Flavin-containing monooxygenases: catalytic mechanism and substrate specificities. Drug Metab. Rev. 19, 1-32.

Ziegler, D.M. 1993. Recent studies on structure and function of multisubstrate flavin-containing monooxygenases. Ann. Rev. Pharmacol. Toxicol. 33, 179-199. 